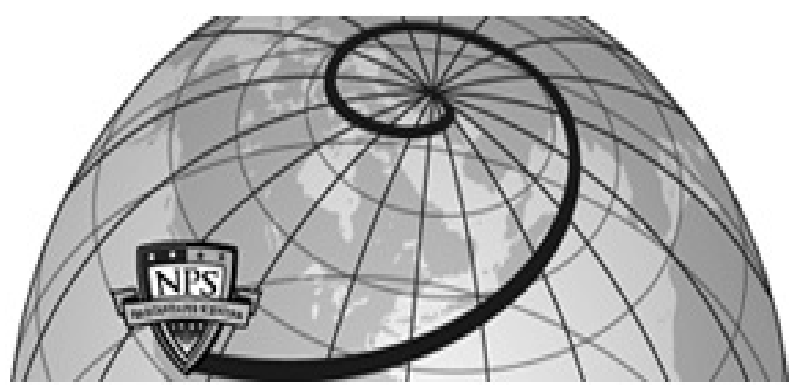

Calhoun: The NPS Institutional Archive DSpace Repository

\title{
Optimization of the ADS Final Turn Maneuver in 2D and 3D
}

Yakimenko, Oleg A.; Slegers, Nathan J.

Monterey, California: Naval Postgraduate School

[Snowflake] Yakimenko, O.A., and Slegers, N.J., Optimization of the ADS Final Turn Maneuver in 2D and 3D, Proceedings of the 21st AIAA Aerodynamic Decelerator Systems Technology Conference, Dublin, Ireland, May 23-26, 2011.

https://hdl.handle.net/10945/35309

This publication is a work of the U.S. Government as defined in Title 17, United States Code, Section 101. Copyright protection is not available for this work in the United States.

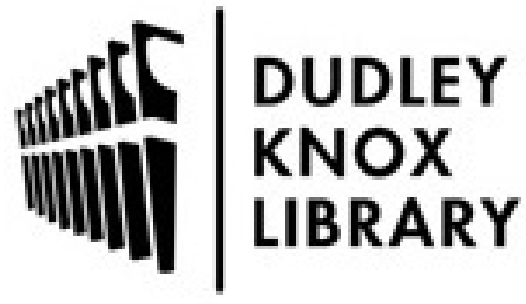

http://www.nps.edu/library
Calhoun is the Naval Postgraduate School's public access digital repository for research materials and institutional publications created by the NPS community. Calhoun is named for Professor of Mathematics Guy K. Calhoun, NPS's first appointed -- and published -- scholarly author.

Dudley Knox Library / Naval Postgraduate School 411 Dyer Road / 1 University Circle Monterey, California USA 93943 


\title{
Optimization of the ADS Final Turn Maneuver in 2D and 3D
}

\author{
Oleg A. Yakimenko* \\ Naval Postgraduate School, Monterey, CA 93943-5107 \\ Nathan J. Slegers ${ }^{\dagger}$ \\ University of Alabama, Huntsville, AL 35899-0266
}

\begin{abstract}
This paper deals with the problem of optimization of the final turn-into-the-wind maneuver of an aerial delivery system with account of the best known winds. The wind model required for the optimization algorithm to work may utilize onboard wind estimates only, incorporate the ground winds provided a priori or on-line by the target ground station, or be based on the winds measured and uplinked by the preceding system. The previous work by the authors took care of the major touchdown error contributor, downwind variation of the winds. The effect of these variations was mitigated by constantly recomputing an optimal reference trajectory to complete a final turn in a given time. This paper presents some modifications of the original optimization routine to accommodate some specific applications including intentional landing with a substantial crosswind component and operating in the mountainous areas with significant variations in the vertical component of the wind (updrafts and downdrafts). Specifically, the paper presents derivation of equations to account for one-, two- and three-dimensional structure of the wind. In addition, adjustments to the optimal control problem using the direct-method-based approach developed earlier for a simple one-dimensional wind model are developed.
\end{abstract}

$\begin{array}{lll} & & \\ \text { ADS } & = & \text { Aerial Delivery System } \\ \text { AGL } & = & \text { above the ground level } \\ \text { BCs } & = & \text { boundary conditions } \\ \text { CEP } & = & \text { Circular Error Probable } \\ \text { FAC } & = & \text { final approach capture (point) } \\ \text { GPS } & = & \text { Global Positioning System } \\ \text { TI } & = & \text { turn initiation (point) } \\ \text { TPBVP } & = & \text { two-point boundary-value problem }\end{array}$

\section{Background}

T $\mathrm{N}$ an attempt to mitigate the effect of unknown variable winds, Slegers and Yakimenko formulated the following 1 two-point boundary-value problem (TPBVP) (Fig.1). ${ }^{1,2}$ Using a right-handed coordinate system $\{W\}$ aligned with the prevailing ground wind (defining a downrange axis) we need to bring a non-powered aerial delivery system (ADS) from some initial point, with the state vector defined at $t=0$ as

$$
\mathbf{x}_{0}=\left[x_{0}, y_{0}, \psi_{0}\right]^{T}
$$

( $x$ - downrange, $y$ - crossrange and $\psi$ - heading in $\{W\}$ ) to another point

$$
\mathbf{x}_{f}=\left[\left(V_{h}^{*}-W\right) T_{a p p}^{d e s}, 0,-\pi\right]^{T}
$$

at $t=t_{f}$. In Eq.(2), $V_{h}^{*}$ is the estimate of a horizontal component of a steady-state airspeed, $W=$ const is the only component of the wind vector

$$
\mathbf{w}=[W, 0,0]^{T}
$$

\footnotetext{
* Professor, Department of Systems Engineering, Code SE/Yk, oayakime@nps.edu, Associate Fellow AIAA.

$\dagger$ Associate Professor, Department of Mechanical and Aerospace Engineering, slegers@mae.uah.edu, Member AIAA.
} 
and $T_{a p p}^{\text {des }}$ is the desired final approach time.

Figure 1 shows the portion of a guided descent to be optimized (appearing in between two vertical lines). It occurs between the turn initiation (TI) point at some altitude $h_{0}$ (defined by the estimate of $W$, and the components of the ADS velocity vector as explained in Ref.1) and final approach capture (FAC) point.

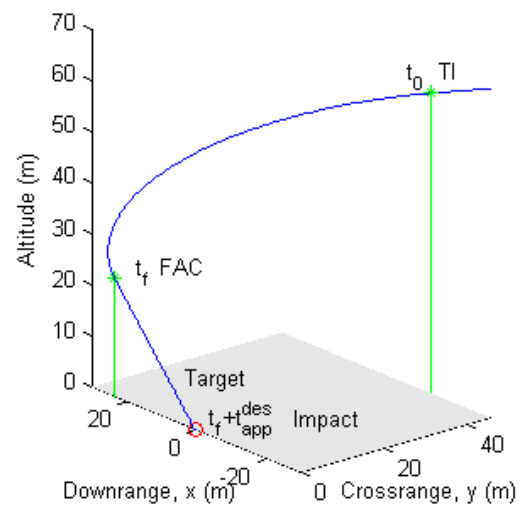

Figure 1. Final turn-into-the-wind maneuver and landing approach.

The altitude at $t=t_{f}$ was defined based on the constant descent rate assumption

$$
h_{f}=V_{v}^{*} T_{a p p}^{d e s}=h_{0}-t_{f} V_{v}^{*}
$$

(here $V_{v}^{*}$ is the estimate of a steady-state descent rate).

Hence, we need to find the trajectory that satisfies the boundary conditions (BCs) (1) and (2) along with the constraint imposed on the control (turn rate), $|\dot{\psi}| \leq \dot{\psi}_{\max }$, and allows completing the maneuver in exactly

$$
T_{\text {turn }}=t_{f}=\frac{h_{0}}{\hat{V}_{v}^{*}}-T_{a p p}^{d e s}
$$

The assumption of a constant descent rate allows eliminating the differential equation for an altitude and reducing ADS' kinematics down to

$$
\left[\begin{array}{c}
\dot{x} \\
\dot{y}
\end{array}\right]=\left[\begin{array}{c}
V_{h}^{*} \cos \psi+W \\
V_{h}^{*} \sin \psi
\end{array}\right]
$$

From these two equations it follows that if the final-turn trajectory is given (defined analytically by $x(t)$ and $y(t))$, then the yaw angle along this trajectory is related to the change of the inertial coordinates as

$$
\psi=\tan ^{-1} \frac{\dot{y}}{\dot{x}-W}
$$

Differentiating Eq.(7) provides with the yaw rate control required to follow the reference final-turn trajectory in a presence of a constant downwind $W$

$$
\dot{\psi}=\frac{\ddot{y}(\dot{x}-W)-\dddot{x} \dot{y}}{(\dot{x}-W)^{2}+\dot{y}^{2}}
$$

Once the TPBVP is solved, Eq.(8) provides the time history of the optimal control $\dot{\psi}_{\text {opt }}(t)$. It is this control profile that is tracked by the ADS' control unit as explained in Ref.1.

The developed guidance and control algorithm was implemented on the Snowflake ADS. ${ }^{3}$ In the period between May of 2008 and May of 2011 this system has been dropped from different deployment platforms from altitudes 2,000-14,000 ft above the ground level (AGL) over 150 times. $^{2}$ During the first set of three drops in May of 2008 the Snowflake ADS exhibited the circular error probable (CEP) of 55m with the standard deviation of $9 \mathrm{~m} .{ }^{4}$ These parameters were gradually reduced to the CEP of $11 \mathrm{~m}$ with the standard deviation of $6 \mathrm{~m}$, exhibited in the set of four drops in August of 2010. ${ }^{4}$ This outstanding performance of the smallest autonomously guided ADS, featuring the cheapest and therefore the worst canopy and being most susceptible to the winds was achieved by implementing the latest technologies in control theory ${ }^{1,6}$ and also by utilizing the best available options of accounting for the unknown surface-layer winds. ${ }^{3,7}$ 
This paper presents modifications of the original optimal control (8) based on alternative wind models, which can be quite different from the one presented in Eq.(3). To this end, the following section presents some flight test data recorded by an onboard sensors suite during a couple of arbitrary chosen drops showing that the actual values of $V_{v}^{*}$ and $W$ can vary quite drastically. Based on observations of these data, Sections III and IV discuss different application-specific modifications of the final-turn optimization routine of Ref.1 and briefly described in the beginning of this section. More precisely, Section III accommodates linear and logarithmic wind profiles in the downwind direction (1D winds optimization), and Section IV considers both downwind and crosswind components (2D optimization). Section V simplifies computational algorithms of Section IV by utilizing the precomputed ballistic winds. Section VI addresses variations in the initial conditions for the final-turn maneuver caused by variable 2D winds. Section VII presents a simple way of mitigation the effects of wind updrafts and downdrafts (3D optimization).

\section{Surface-Layer Winds}

Figure 2 presents two samples of data measured/estimated and recorded onboard the Snowflake ADS. The plots in the first column belong to one drop terminated with the miss distance of $10 \mathrm{~m}$, and in the second column - to a second drop that resulted in a miss distance of $6 \mathrm{~m}$. The first row of the plots presents the altitude versus time profile. The second row shows estimated downwind component of the wind (which changes all the time based on the latest observations of the ground track speed measured by the onboard Global Positioning System (GPS) receiver). The third row shows vertical speed of the ADS as measured by GPS and smoothed (filtered) by the onboard inertial navigation unit. The last row of the plots presents different stages of flight when these data were collected.
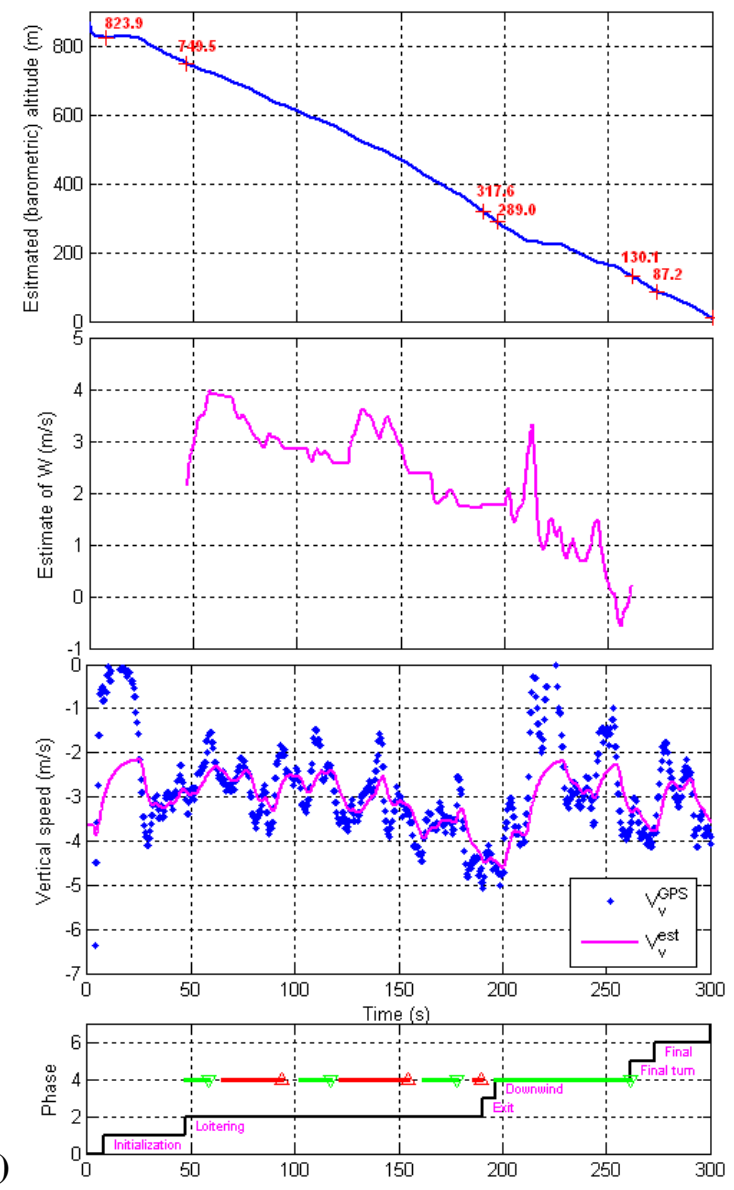
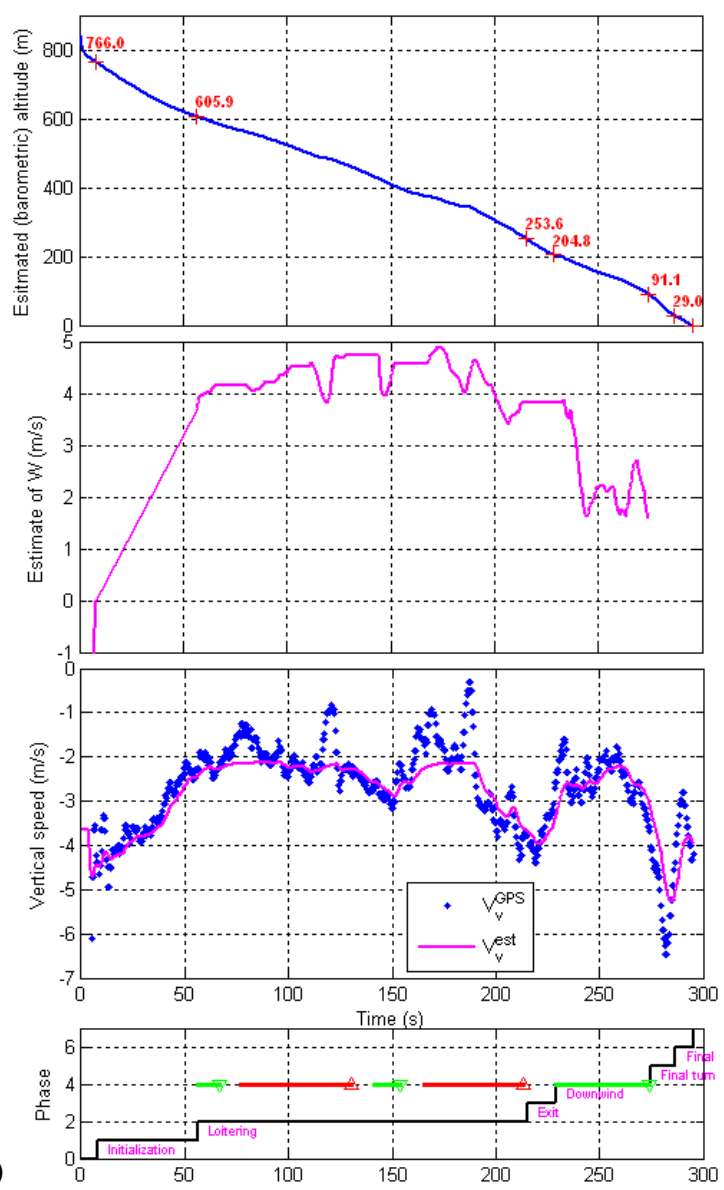

Figure 2. Flight parameters recorded during the two drops in August of 2010.

As seen in practice, neither $V_{v}^{*}$ nor $W$ used in equation of Section 1 are constant. While the first set of data exhibits a kind of gradual decrease of the downwind component $W$ with time (altitude), the second set of data features more or less constant winds up to about 200m altitude AGL with a sudden halved decrease below this 
altitude. For convenience of analysis, Fig.3 presents the values of $V_{v}^{*}$ and $W$ for the same sets of data as versus altitude, rather than time. Looking at both sets of data together one can notice that the descent rate (negative of the vertical speed) varies from $0 \mathrm{~m} / \mathrm{s}$ (as a result of some updraft motion) all way up to $6 \mathrm{~m} / \mathrm{s}$, apparently affected by a close to the ground downdraft. It is indicative that both sets of data belong to the same ADS dropped at the same location less than an hour apart. No wonder that parafoils systems, being influenced by varying winds exhibit inconstant performance.

a)

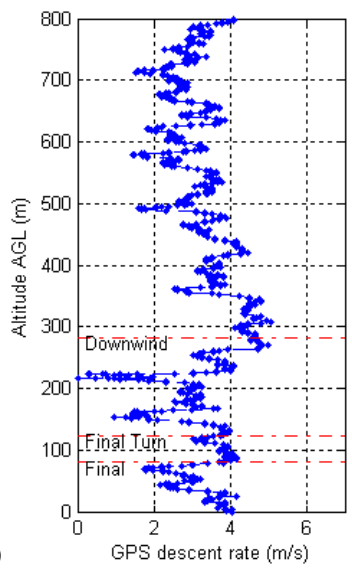

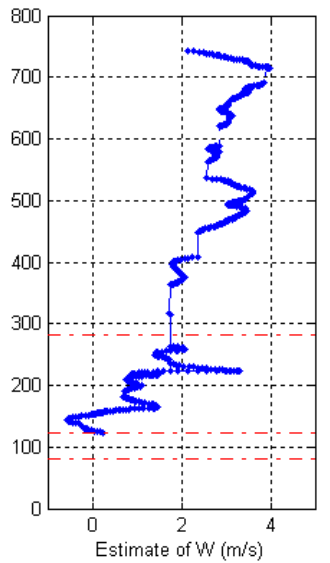

Estimate of $W(\mathrm{~m} / \mathrm{s})$

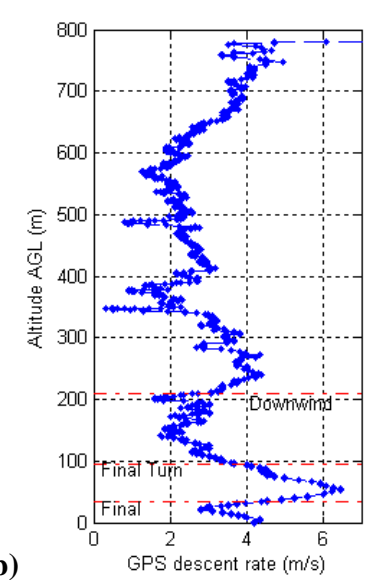

b)

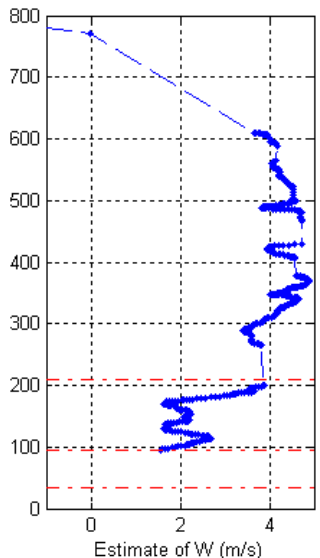

Figure 3. Altitude dependences of the ADS estimates.

According to the guidance strategy described in Ref.1 the winds aloft only affect the location of the computed TI point along the downwind leg. This leaves all unmodeled dynamics to be handled at the following stages of flight, i.e. final (base) turn and final landing approach. Data presented in Figs.2 and 3 is zoomed to the final stages in Figs. 4 and 5 to show parameter variations at the surface layer, staring at about $300 \mathrm{~m}$ to include the downwind leg where the decision to turn is made.

Obviously, in the general case the unaccounted winds may have components in all three directions

$$
\mathbf{w}^{\text {dist }}(h)=\left[w_{x}, w_{y}, w_{z}\right]^{T}
$$

(here $w_{x}$ denotes a downwind component, not accounted for by Eq.(3), while $w_{z}$ is considered positive for downdrafts to be consistent with the descent rate sign convention). With disturbances (9) the kinematic equations (6) become three-dimensional

$$
\left[\begin{array}{c}
\dot{x} \\
\dot{y} \\
\dot{h}
\end{array}\right]=\left[\begin{array}{c}
V_{h}^{*} \cos \psi+W+w_{x} \\
V_{h}^{*} \sin \psi+w_{y} \\
-V_{v}^{*}-w_{z}
\end{array}\right]
$$

In the original algorithm of Ref.1 however the unaccounted winds (9) were treated as disturbances, so that it was up to the control system to mitigate their effect while still using Eq.(6) to compute the reference control (7). Surely, these disturbances were then the primary reason for the ADS not tracking the calculated optimal-turn trajectory precisely. As shown in numerous simulations and in practice it is these winds that can cause the ADS to land short of the target (in the case of the higher than expected head winds) or overshoot it (tail winds). That is why the optimal trajectory needs to be constantly updated during the final turn, each time starting from the current (off the original trajectory) initial conditions (IC) and still forcing the ADS to be at point (3) within an updated $T_{\text {turn }}$ (5).

The goal of the following sections is to account for wind disturbances (9) at the stage of generating the reference control, i.e. trying to use Eq.(10) instead of Eq.(6). Obviously, it can be done only if the wind disturbance components (9) can be modeled (using more information about the winds known a priori). To this end, Section III starts with more accurate modeling of downwind component of the surface winds, followed by Section IV introducing a crosswind component and ending with Section V discussing the vertical wind component.

\section{Optimization Based on the Linear and Logarithmic Surface-Layer Wind Models}

Assume that instead of a constant $x$-component of the prevailing wind $W$ versus altitude $h$ (Eq.(2)) we have a linear profile

$$
W(h)=W_{G}+K h
$$


where $W_{G}$ is a known ground wind and coefficient $K=\left(W_{0}-W_{G}\right) h_{0}^{-1}$ is defined by the ground wind $W_{G}$ and wind $W_{0}$ measured at an altitude $h_{0}$ (corresponding to the point where the final turn begins). In terms of Eq.(9) it means that we are trying to model the downwind disturbance as $w_{x}(h)=W_{G}-W_{0}+K h$. Such a profile might be based on the known ground winds (available from the nearby airport, measured by the target ground station, ${ }^{3,7}$ etc.) uplinked to the descending ADS.
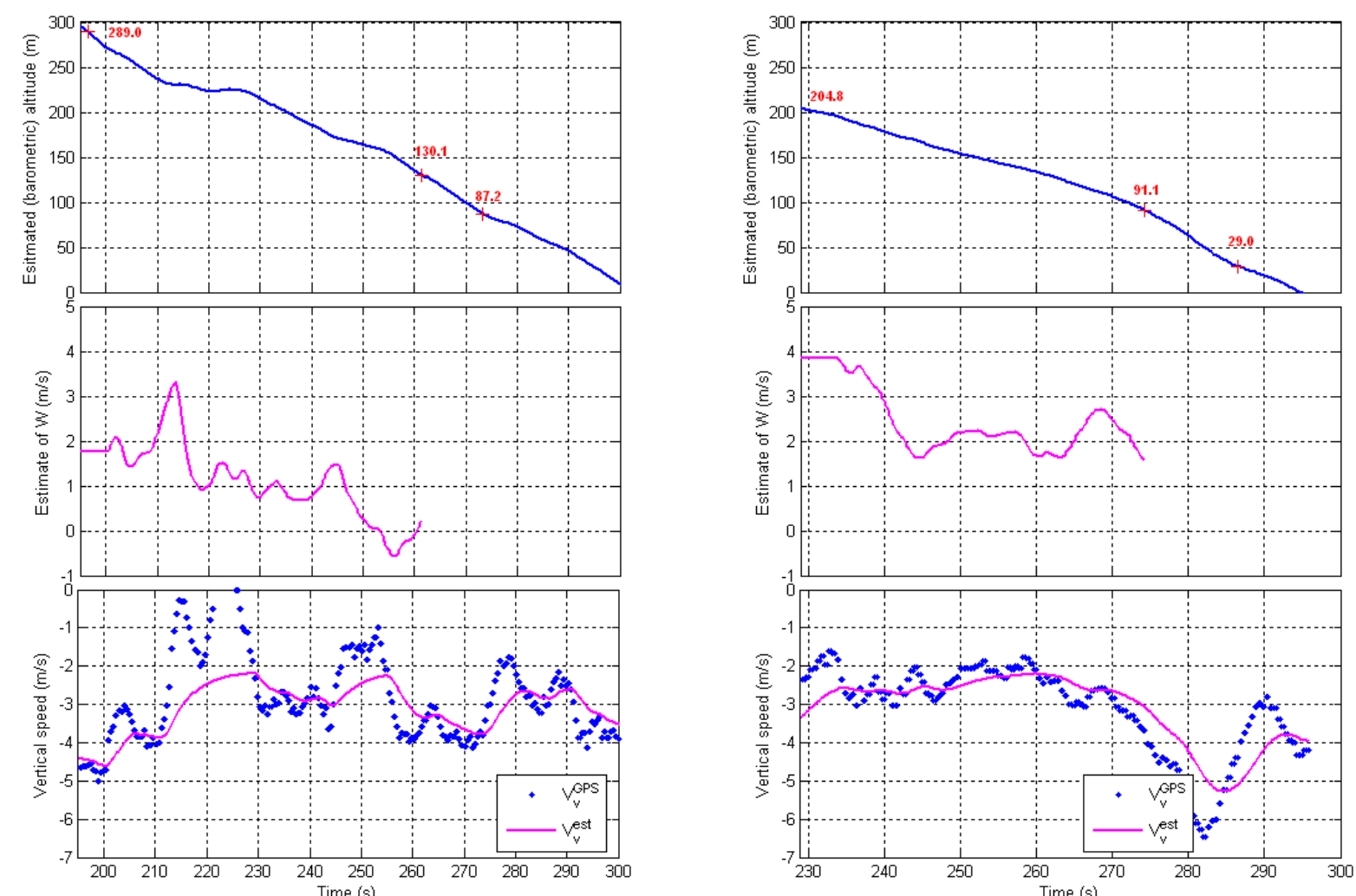

a)

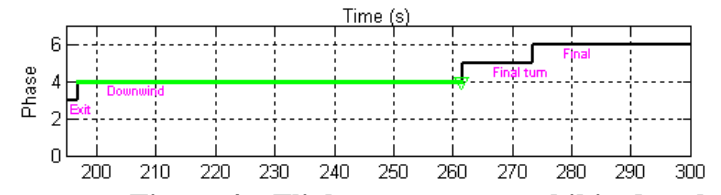

b)
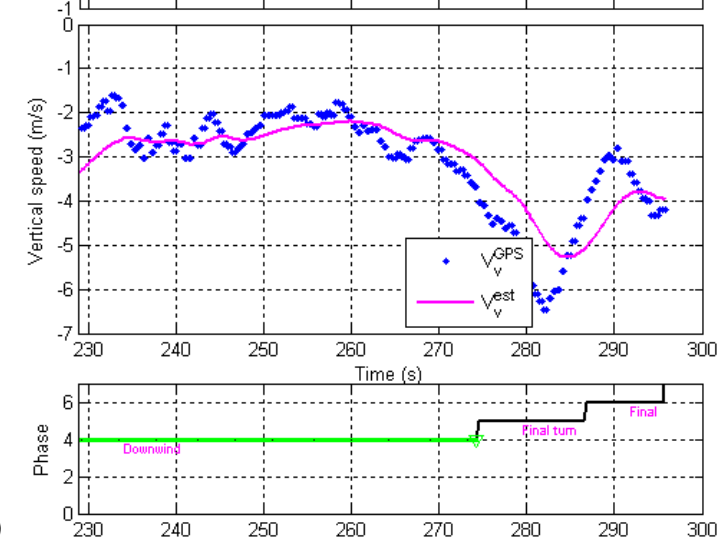

Figure 4. Flight parameters exhibited at the 300m surface-layer (zoomed-in versions of Fig.2).
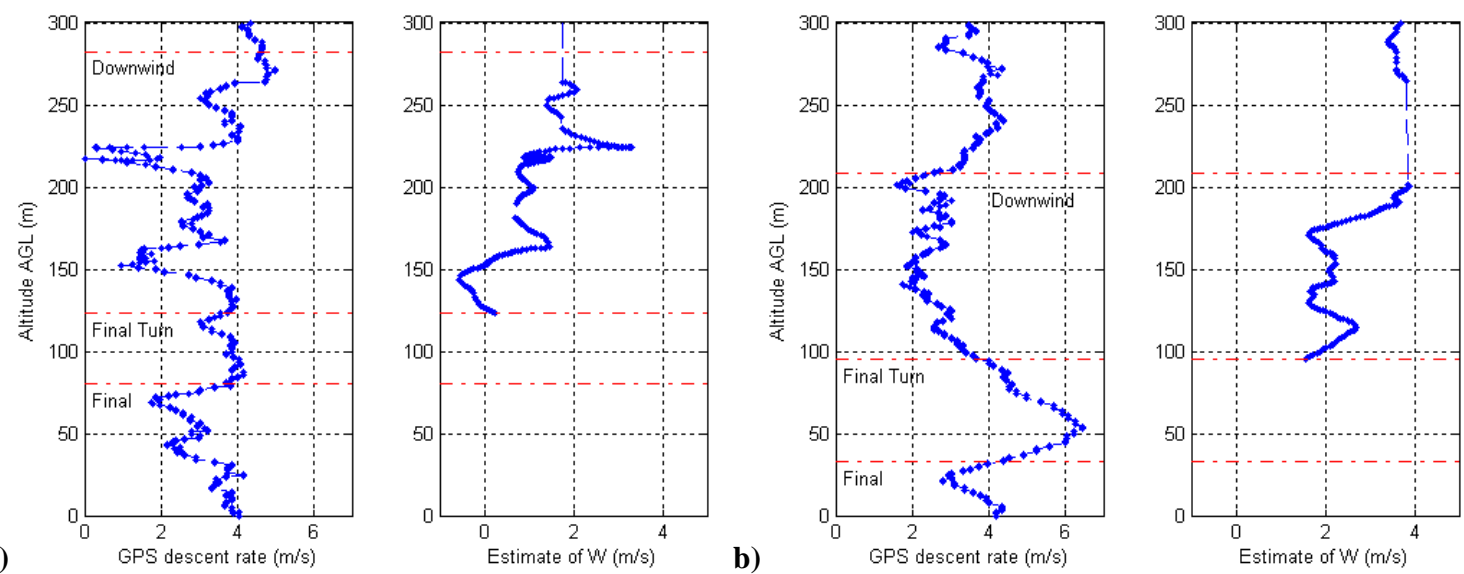

Figure 5. Altitude dependences of the ADS' estimates between the surface and 300m AGL altitude.

Then, the original TPBVP of Section I should be reformulated for a slightly different system of kinematic equations 


$$
\left[\begin{array}{c}
\dot{x} \\
\dot{y}
\end{array}\right]=\left[\begin{array}{c}
V_{h}^{*} \cos \psi+W(h) \\
V_{h}^{*} \sin \psi
\end{array}\right]
$$

and different BCs. To be more specific, starting from some (different) initial point, defined by a different expression for a distance past the target on the downwind leg to initiate the final turn maneuver (defined in Ref.1 as $D_{\text {switch }}$ ), we will need to bring a parafoil to the point

$$
\mathbf{x}_{f}=\left[\left(V_{h}^{*}-W\right) T_{a p p}^{d e s}-\frac{1}{2} K V_{v}^{*}\left(T_{a p p}^{d e s}\right)^{2}, 0,-\pi\right]^{T}
$$

(computation of $D_{\text {switch }}$ will be addressed in Section VI).

To compute the offset in Eq.(13) we used the fact that the final landing approach starts at the altitude $V_{v}^{*} T_{a p p}^{\text {des }}$, so that using an obvious relation

$$
d t=-\frac{d h}{V_{v}^{*}}
$$

we may write

$$
x_{f}=\int_{0}^{T_{a p p}^{d e s}}\left(V_{h}^{*}-W\right) d t=\int_{V_{v}^{*} T_{a p p}^{d e s}}^{0}\left(V_{h}^{*}-W\right) \frac{-d h}{V_{v}^{*}}=\int_{0}^{V_{v}^{*} \text { app }}\left(V_{h}^{*}-W\right) \frac{d h}{V_{v}^{*}}=\left(V_{h}^{*}-W_{G}\right) T_{a p p}^{d e s}-\frac{1}{2} K V_{v}^{*}\left(T_{a p p}^{d e s}\right)^{2}
$$

In this case, inverting equations (12) yields

$$
\psi=\tan ^{-1} \frac{\dot{y}}{\dot{x}-W(h)}
$$

Compared to Eq.(7), Eq.(8) features and altitude-dependent wind profile $W(h)$, so that its differentiation with account of Eq.(11) results in a slightly different equation for the turn rate

$$
\dot{\psi}=\frac{\ddot{y}(\dot{x}-W)-(\ddot{x}-\dot{W}) \dot{y}}{(\dot{x}-W)^{2}+\dot{y}^{2}}=\frac{\ddot{y}(\dot{x}-W)-\left(\ddot{x}-K V_{v}^{*}\right) \dot{y}}{(\dot{x}-W)^{2}+\dot{y}^{2}}
$$

(cf. with Eq.(8)).

The only modifications the numerical algorithm described in Ref.1 requires in this case is that it should involve the new BCs

$$
\begin{array}{ll}
{\left[\begin{array}{c}
\dot{x} \\
\dot{y}
\end{array}\right]_{\tau=0}=\left[\begin{array}{c}
V_{h}^{*} \cos \psi_{0}+W_{0} \\
V_{h}^{*} \sin \psi_{0}
\end{array}\right],} & {\left[\begin{array}{c}
\ddot{x} \\
\ddot{y}
\end{array}\right]_{\tau=0}=\left[\begin{array}{c}
K V_{v}^{*}+\dot{\psi}_{0} V_{h}^{*} \sin \psi_{0} \\
\dot{\psi}_{0} V_{h}^{*} \cos \psi_{0}
\end{array}\right],} \\
{\left[\begin{array}{c}
x \\
y
\end{array}\right]_{\tau=\tau_{f}}=\left[\begin{array}{c}
\left(V_{h}^{*}-W_{G}\right) T_{a p p}^{\text {des }}-\frac{1}{2} K V_{v}^{*}\left(T_{a p p}^{\text {des }}\right)^{2} \\
0
\end{array}\right], \quad\left[\begin{array}{c}
\dot{x} \\
\dot{y}
\end{array}\right]_{\tau=\tau_{f}}=\left[\begin{array}{c}
-V_{h}^{*}+W_{G}+K V_{v}^{*} T_{a p p}^{d e s} \\
0
\end{array}\right]}
\end{array}
$$

as well as computation of an altitude

$$
h_{j}=h_{j-1}-V_{v}^{*} \Delta t_{j-1}, j=2, \ldots, N,\left(h_{1} \equiv h_{0}\right)
$$

and the corresponding wind magnitude at each computational node

$$
W_{j}=W_{G}+K h_{j}
$$

The latter two values then are to be used to compute time intervals between two computational nodes

$$
\Delta t_{j-1}=\sqrt{\frac{\left(x_{j}-x_{j-1}\right)^{2}+\left(y_{j}-y_{j-1}\right)^{2}}{V_{h}^{* 2}+0.25\left(W_{j}+W_{j-1}\right)^{2}+V_{h}^{*}\left(W_{j}+W_{j-1}\right) \cos \psi_{j-1}}}
$$

and heading

$$
\psi_{j}=\tan ^{-1} \frac{\lambda_{j} y_{j}^{\prime}}{\lambda_{j} x_{j}^{\prime}-W_{j}}
$$

In the case when the ground winds are not available a general logarithmic wind profile may be used in lieu of the liner profile of Eq.(11) ${ }^{8}$

$$
W(h)=\beta+a \ln (h)
$$

In this case some of the above equations will be replaced with the new ones 


$$
\begin{gathered}
x_{f}=\int_{0}^{V_{v}^{*} \text { dapp }}\left(V_{h}^{*}-W\right) \frac{d h}{V_{v}^{*}}=\left(V_{h}^{*}+\alpha-\beta\right) T_{\text {app }}^{\text {des }}-\alpha T_{\text {app }}^{d e s} \ln \left(V_{v}^{*} T_{a p p}^{d e s}\right) \\
{\left[\begin{array}{c}
\ddot{x} \\
\ddot{y}
\end{array}\right]_{\tau=0}=\left[\begin{array}{c}
-\dot{\psi}_{0} V_{h}^{*} \sin \psi_{0}-a h_{0}^{-1} V_{v}^{*} \\
\dot{\psi} V_{h}^{*} \cos \psi_{0}
\end{array}\right]} \\
{\left[\begin{array}{l}
x \\
y
\end{array}\right]_{\tau=\tau_{f}}=\left[\begin{array}{c}
\left(V_{h}^{*}+\alpha-\beta\right) T_{a p p}^{d e s}-\alpha T_{a p p}^{d e s} \ln \left(V_{v}^{*} T_{a p p}^{d e s}\right) \\
0
\end{array}\right], \quad\left[\begin{array}{c}
\dot{x} \\
\dot{y}
\end{array}\right]_{\tau=\tau_{f}}=\left[\begin{array}{c}
-V_{h}^{*}+\beta+a \ln \left(V_{v}^{*} T_{a p p}^{d e s}\right) \\
0
\end{array}\right]}
\end{gathered}
$$

and

$$
W_{j}=\beta+a \ln \left(h_{j}\right)
$$

The remaining equations will still be the same.

\section{Accommodating Cross-Wind Data}

The optimization routine of the Snowflake guidance algorithm can also accommodate crosswinds if they are known in one form or another. This capability may be useful in organizing a swarm attack or landing onto a ship's deck, not necessarily aligned with the wind. ${ }^{9}$ Consider

$$
w_{x}(h)=f_{x}(h), \quad w_{y}(h)=f_{y}(h)
$$

to be $x$ - (downwind) and $y$ - (crosswind) components of a horizontal wind profile approximated with some analytical dependence (e.g. of the form of Eq.(11) or (24) or cubic spline). Then, we can write

$$
\left[\begin{array}{c}
\dot{x} \\
\dot{y}
\end{array}\right]=\left[\begin{array}{l}
V_{h}^{*} \cos \psi+w_{x}(h) \\
V_{h}^{*} \sin \psi+w_{y}(h)
\end{array}\right]
$$

Note that instead of Eq.(6) we are now using the first two equations of (10), emphasizing that the entire trajectory is intentionally aligned not with the major wind component, so that $w_{y}(h)$ can actually be even larger than $w_{x}(h)$ (i.e. we let $W \equiv 0$ ).

Accounting for the new kinematics described by Eq.(30) the final point can be defined as

$$
\mathbf{x}_{f}=\left[x_{f}, y_{f},-\pi\right]^{T}
$$

where the offsets in $x$ - and $y$ - direction will be computed as

$$
x_{f}=V_{h}^{*} T_{a p p}^{d e s}-\int_{0}^{V_{v}^{*} T_{a p p}^{d e s}} w_{x}(h) \frac{d h}{V_{v}^{*}}, \quad y_{f}=-\int_{0}^{V_{v}^{*} T_{a p p}^{d e s}} w_{y}(h) \frac{d h}{V_{v}^{*}}
$$

The heading angle equation will be

$$
\psi=\tan ^{-1} \frac{\dot{y}-w_{y}(h)}{\dot{x}-w_{x}(h)}
$$

while its derivative will be presented by

$$
\dot{\psi}=\frac{\left(\ddot{y}-w_{y}^{\prime}(h) V_{v}^{*}\right)\left(\dot{x}-w_{x}(h)\right)-\left(\ddot{x}-w_{x}^{\prime}(h) V_{v}^{*}\right)\left(\dot{y}-w_{y}(h)\right)}{\left(\dot{x}-w_{x}(h)\right)^{2}+\left(\dot{y}-w_{y}(h)\right)^{2}}
$$

(where $w_{x}^{\prime}=d w_{x} / d h$ and $w_{y}^{\prime}=d w_{y} / d h$ ). The total speed will now be expressed as

$$
\left|V_{G}\right|=\sqrt{\dot{x}^{2}+\dot{y}^{2}}=\sqrt{V_{h}^{* 2}+\left(w_{x}(h)+w_{y}(h)\right)^{2}+2 V_{h}^{*}\left(w_{x}(h) \cos \psi+w_{y}(h) \sin \psi\right)}
$$

The numerical procedure will proceed with the boundary conditions

$$
\begin{gathered}
{\left[\begin{array}{l}
x \\
y
\end{array}\right]_{\tau=0}=\left[\begin{array}{l}
x_{0} \\
y_{0}
\end{array}\right], \quad\left[\begin{array}{l}
\dot{x} \\
\dot{y}
\end{array}\right]_{\tau=0}=\left[\begin{array}{l}
V_{h}^{*} \cos \psi_{0}+w_{x}\left(h_{0}\right) \\
V_{h}^{*} \sin \psi_{0}+w_{y}\left(h_{0}\right)
\end{array}\right], \quad\left[\begin{array}{c}
\ddot{x} \\
\ddot{y}
\end{array}\right]_{\tau=0}=\left[\begin{array}{c}
-\dot{\psi}_{0} V_{h}^{*} \sin \psi_{0}+w_{x}^{\prime}(h) V_{y}^{*} \\
\dot{\psi}_{0} V_{h}^{*} \cos \psi_{0}+w_{y}^{\prime}(h) V_{y}^{*}
\end{array}\right]} \\
{\left[\begin{array}{l}
x \\
y
\end{array}\right]_{\tau=\tau_{f}}=\left[\begin{array}{l}
x_{f} \\
y_{f}
\end{array}\right], \quad\left[\begin{array}{c}
\dot{x} \\
\dot{y}
\end{array}\right]_{\tau=\tau_{f}}=\left[\begin{array}{c}
-V_{h}^{*}+w_{x}(h) \\
w_{y}(h)
\end{array}\right], \quad\left[\begin{array}{c}
\ddot{x} \\
\ddot{y}
\end{array}\right]_{\tau=\tau_{f}}=\left[\begin{array}{l}
0 \\
0
\end{array}\right]}
\end{gathered}
$$


and will involve computing wind components at each step

$$
w_{x j}=f_{x}\left(h_{j}\right) \quad \text { and } \quad w_{y j}=f_{y}\left(h_{j}\right)
$$

to be used in the numerical equations similar to those given in Section IV.

\section{Using Ballistic Winds}

In the previous section we were relying on some analytical wind profiles $w_{x}=f_{x}(h)$ and $w_{y}=f_{y}(h)$. In practice however the components of the horizontal wind can be available as the look-up tables, containing triples $h_{j}$, $w_{x j}$, and $w_{y j}$ (or wind magnitude and direction, instead of the last two parameters). In this case, to avoid computing derivatives $w_{x}^{\prime}$ and $w_{y}^{\prime}$ in Eqs. (34) and (36) we could use the so-called ballistic winds. By definition, if at some altitude $H$ we have a ballistic wind of magnitude $W_{B}$ and direction $\Psi_{W}$, then the effect of variable winds $w_{x}(h)$ and $w_{y}(h)$ for some system with the descent rate $V_{v}$ on its way from altitude $H$ down to the surface is reduced to simple formulas

or in other words

$$
x(h)=\frac{H}{V_{v}^{*}} W_{B} \cos \Psi_{W}, \quad y(h)=\frac{H}{V_{v}^{*}} W_{B} \sin \Psi_{W}
$$

$$
\int_{0}^{H} \frac{1}{V_{v}^{*}} w_{x}(h) d h=\frac{H}{V_{v}^{*}} W_{B} \cos \Psi_{W}, \quad \int_{0}^{H} \frac{1}{V_{v}^{*}} w_{y}(h) d h=\frac{H}{V_{v}^{*}} W_{B} \sin \Psi_{W}
$$

(Note, usually $w_{x}$ and $w_{y}$ are measured in the local North-East-Down coordinate system, so $\Psi_{W}$ is a direction with respect to the true North. However in our case $w_{x}$ and $w_{y}$ are expected to be provided by the first descending system in $\{W\}$, so that $\Psi_{W}$ will also be calculated in $\{W\}$ ).

Substituting definite integrals in Eq.(40) with the finite sum of trapezoids based on the discrete values of $h_{k}, w_{k}$ and $\psi_{w k}, k=1, \ldots, M$, we get

$$
\sum_{k=2}^{M}\left(h_{k}-h_{k-1}\right) \frac{w_{x k}+w_{x, k-1}}{2}=h_{M} W_{M} \cos \Psi_{W ; M}, \quad \sum_{k=2}^{M}\left(h_{k}-h_{k-1}\right) \frac{w_{y k}+w_{y, k-1}}{2}=h_{M} W_{M} \sin \Psi_{W ; M}
$$

The index starts from 2 because by definition the winds measurements at the lowest altitude can be considered ballistic winds at this altitude.

From Eqs.(41) it further follows that

$$
\begin{aligned}
\tan \Psi_{W ; M} & =\frac{\sum_{k=2}^{M}\left(h_{k}-h_{k-1}\right)\left(w_{y k}+w_{y, k-1}\right)}{\sum_{k=2}^{M}\left(h_{k}-h_{k-1}\right)\left(w_{x k}+w_{x, k-1}\right)} \\
W_{M}= & \frac{1}{2 h_{M}} \sqrt{\left(\sum_{k=2}^{M}\left(h_{k}-h_{k-1}\right)\left(w_{x k}+w_{x, k-1}\right)\right)^{2}+\left(\sum_{k=2}^{M}\left(h_{k}-h_{k-1}\right)\left(w_{y k}+w_{y, k-1}\right)\right)^{2}}
\end{aligned}
$$

For the specific case when $h_{k}-h_{k-1}=\Delta h=$ const $, k=2, \ldots, M$, Eqs.(42) can be further reduced to

$$
\tan \Psi_{W ; M}=\frac{\sum_{k=2}^{M}\left(w_{y k}+w_{y, k-1}\right)}{\sum_{k=2}^{M}\left(w_{x k}+w_{x, k-1}\right)}, \quad W_{M}=\frac{\Delta h}{2 h_{M}} \sqrt{\left(\sum_{k=2}^{M}\left(w_{x k}+w_{x, k-1}\right)\right)^{2}+\left(\sum_{k=2}^{M}\left(w_{y k}+w_{y, k-1}\right)\right)^{2}}
$$

If the ballistic winds are known a priori, meaning that $w_{x}=f_{x}(h)$ and $w_{y}=f_{y}(h)$ were provided by the first descended system as a look-up table, then the original guidance algorithm of Ref.1 assuming constant winds, can be used with no change. 


\section{Computation of the Initial Conditions for the Final into-the Wind Turn}

In addition to the modifications of the original algorithm of Ref.1 discussed in the previous section, different wind models require changing the initial conditions to initiate the final turn as well. Following the original guidance algorithm of Ref.1, the $x$ - (downwind direction) budget equation for two phases, base turn $\left(t \in\left[t_{0} ; t_{1}\right]\right)$ and final approach $\left(t \in\left[t_{1} ; t_{2}\right]\right)$ can be represented as

$$
D_{\text {switch }}=-\int_{t_{0}}^{t_{1}} W(h) d t+\int_{t_{1}}^{t_{2}}\left(V_{h}^{*}-W(h)\right) d t=V_{h}^{*} T_{\text {app }}-\int_{t_{0}}^{t_{2}} W(h) d t=V_{h}^{*} T_{\text {app }}-\left.\left(T_{\text {turn }}+T_{\text {app }}\right) \bar{W}\right|_{h_{0}} ^{0}
$$

Here $D_{\text {switch }}$ is the distance passed the target's traverse when the base turn should be initiated (in this case upon completion of the aforementioned two phases the ADS will be right on/above the target), and $\left.\bar{W}\right|_{h_{0}} ^{0}$ denotes a downwind component of the wind averaged within the altitude range $h \in\left[0 ; h_{0}\right]$.

The altitude budget equation for these two plus the portion of the downwind leg phase starting at some altitude $h$ at a distance $x$ from the target's traverse is

$$
h=V_{v}^{*}\left(\frac{-x+D_{\text {switch }}}{V_{h}^{*}+\left.\bar{W}\right|_{h} ^{h_{0}}}\right)+V_{v}^{*} T_{\text {turn }}+V_{v}^{*} T_{a p p}
$$

Ideally, when $x=D_{\text {switch }}$ the altitude $h$ should be equal to $V_{v}^{*}\left(T_{\text {turn }}+T_{a p p}\right)$. In practice however it may not be the case. In order to eliminate the altitude error, we may want to adjust the actual final approach time $T_{a p p}$.

Resolving Eqs. (45) and (45) with respect to $D_{\text {switch }}$ and $T_{\text {app }}$ yields

$$
\begin{gathered}
D_{\text {switch }}=\frac{h V_{h}^{*}\left(V_{h}^{*}-\left.\bar{W}\right|_{h_{0}} ^{0}+\left.\bar{W}\right|_{h} ^{0}\right)+\left.h \bar{W}\right|_{h_{0}} ^{0}\left(\left.\bar{W}\right|_{h_{0}} ^{0}-\left.\bar{W}\right|_{h} ^{0}\right)}{V_{v}^{*}\left(2 V_{h}^{*}-\left.2 \bar{W}\right|_{h_{0}} ^{0}+\left.\bar{W}\right|_{h} ^{0}\right)}-\frac{-x\left(V_{h}^{*}+\left.\bar{W}\right|_{h_{0}} ^{0}\right)+T_{\text {turn }} V_{h}^{*}\left(V_{h}^{*}-\left.\bar{W}\right|_{h_{0}} ^{0}+\left.\bar{W}\right|_{h} ^{0}\right)}{2 V_{h}^{*}-\left.2 \bar{W}\right|_{h_{0}} ^{0}+\left.\bar{W}\right|_{h} ^{0}} \\
T_{\text {app }}=\frac{h\left(V_{h}^{*}-\left.\bar{W}\right|_{h_{0}} ^{0}+\left.\bar{W}\right|_{h} ^{0}\right)}{V_{v}^{*}\left(2 V_{h}^{*}-\left.2 \bar{W}\right|_{h_{0}} ^{0}+\left.\bar{W}\right|_{h} ^{0}\right)}-\frac{-x+T_{\text {turn }}\left(V_{h}^{*}-\left.2 \bar{W}\right|_{h_{0}} ^{0}+\left.\bar{W}\right|_{h} ^{0}\right)}{2 V_{h}^{*}-\left.2 \bar{W}\right|_{h_{0}} ^{0}+\left.\bar{W}\right|_{h} ^{0}}
\end{gathered}
$$

(here we used an obvious relation $\left.\bar{W}\right|_{h} ^{h_{0}}=\left.\bar{W}\right|_{h} ^{0}-\left.\bar{W}\right|_{h_{0}} ^{0}$ ).

In the case of $W=\left.\bar{W}\right|_{h} ^{0}=\left.\bar{W}\right|_{h_{0}} ^{0}=$ const $(h)$, Eqs. (46) and (47) are simplified to those of the original guidance algorithm of Ref.1. In all other cases, Eqs. (46) and (47) have to be used. For the linear and logarithmic surface-layer wind models (12) and (24) of Section III the averaged winds from some current altitude $h$ down to the ground can be computed as

$$
\begin{gathered}
\left.\bar{W}\right|_{h} ^{0}=\frac{1}{h} \int_{0}^{h}\left(W_{G}+K h\right) d h=W_{G}+\frac{1}{2} K h \\
\left.\bar{W}\right|_{h} ^{0}=\frac{1}{h} \int_{0}^{h}(\beta+a \ln (h)) d h=\beta+\alpha \ln (h)-\alpha
\end{gathered}
$$

respectively. Substituting $\left.\bar{W}\right|_{h} ^{0}$ and $\left.\bar{W}\right|_{h_{0}} ^{0}$, computed using Eq.(48) or Eq.(49), into Eqs.(46),(47) results in in a windmodel-specific values for $D_{\text {switch }}$ and $T_{a p p}$ (they are quite bulky and are not given here).

Alternatively, the values for $\left.\bar{W}\right|_{h} ^{0}$ and $\left.\bar{W}\right|_{h_{0}} ^{0}$ can be substituted with the corresponding downrange component of the ballistic winds (computed in accordance with Eq.(42) for $h$ and $h_{0}$, respectively) 


$$
\left.\bar{W}\right|_{h} ^{0}=W_{B}(h) \cos \left(\Psi_{W}(h)\right),\left.\quad \bar{W}\right|_{h_{0}} ^{0}=W_{B}\left(h_{0}\right) \cos \left(\Psi_{W}\left(h_{0}\right)\right)
$$

In the case of the two-dimensional wind model (including the crosswind component), Eqs.(46)-(50) will still be valid, since computation of $D_{\text {switch }}$ relies on the downrange winds only.

\section{Accounting for Vertical Wind Disturbances}

Suppose we managed to have two ADSs, so that while descending the first one produces and passes the winds estimates to the second one. Such estimates will be represented by the GPS time-stamped quadruples: $h_{k}, w_{x k}, w_{y k}$, and $w_{z k}, k=2,3, \ldots, M$ ( $k=1$ corresponds to $\left.h=0\right)$. As shown in the previous sections, even if triplets $h_{k}, w_{x k}$, $w_{y k}$ are available, accounting for these data while generating a reference trajectory may still pose a computational problem. Accounting for the vertical component of the wind $w_{z}(h)$, i.e. updrafts and downdrafts, is even more complicated. In a non-stable atmosphere this component of the wind may cause the same type of problem as unaccounted for horizontal winds simply because it changes the descent time forcing ADS to land sooner (shorter of the target) and later (resulting in the overshoot).

For example, consider a sudden updraft on the downwind leg (Figs.4a and 5a) or downdraft while ADS is at the final turn (Figs.4b and 5b). Obviously, such events may cause a serious problem. At the downwind leg a vertical motion of the air mass cause a violation of the altitude budget equation (45). The final turn maneuver is therefore the last chance to mitigate this violation. However, updrafts and downdrafts at this phase of descent mess the optimal solution obtained assuming a certain time of maneuver $T_{\text {turn }}$ (Eq.(5)). The capability to recompute this maneuver while turning even at each control cycle (if needed) was a real breakthrough of the original guidance algorithm. ${ }^{1}$ Yet, as opposed to updrafts, downdrafts may be still of the major concern because they may decrease the time of the final-turn maneuver at once, leaving no time to recover. Hence, accounting to the vertical winds may be quite beneficial.

Suppose that the vertical component of the wind is known. Again, it most likely comes as a look-up table, $h_{k}$ vs. $w_{z k}$, but theoretically we could use a low-order polynomial regression to approximate it with some analytical dependence $w_{z}(h)$. In this case this dependence may be used to modify the vertical motion equation (14) to

$$
d t=-\frac{d h}{V_{v}^{*}+w_{z}(h)}
$$

This equation is then to be used in Eqs. (15), (25), (32), (40), (45), (48), and (49). Obviously, depending on the specific analytical representation $w_{z}(h)$ the resulting equations may be very bulky, so the alternative approach may be based on the analogous of the ballistic winds concept introduced in Section $\mathrm{V}$, which does not require analytical regression but can rather utilize $\left(h_{k}, w_{z k}\right)$ pairs explicitly.

Following Eqs. (40) and (41), let us introduce

$$
\left.\bar{w}_{z}\right|_{h} ^{0}=\frac{1}{h} \int_{0}^{h} w_{z}(h) d h \approx \frac{1}{2 h} \sum_{k=2}^{M}\left(h_{k}-h_{k-1}\right)\left(w_{z k}+w_{z, k-1}\right)
$$

which denotes a downdraft component of the wind averaged within the altitude range $h \in[0 ; h]$. Using this average downdraft we can rewrite Eq.(51) as

$$
h=T\left(V_{v}^{*}+\left.\bar{w}_{z}\right|_{h} ^{0}\right)
$$

This equation (implicit in $h$ ) allows you to estimate time $T$ needed to descent from the altitude $h$. Now let us use Eq.(53) to correct Eq.(5). To this end let us use Eq.(53) to replace Eq.(4) with

$$
\left(V_{v}^{*}+\left.\bar{w}_{z}\right|_{h_{F A}} ^{0}\right) T_{a p p}^{d e s}=h_{0}-T_{\text {turn }}\left(V_{v}^{*}+\left.\bar{w}_{z}\right|_{h_{T I}} ^{h_{F A}}\right)
$$

Noting that $\left.\bar{w}_{z}\right|_{h_{T I}} ^{h_{F A}}=\left.\bar{w}_{z}\right|_{h_{I I}} ^{0}-\left.\bar{w}_{z}\right|_{h_{F A}} ^{0}$ we arrive to

$$
T_{\text {turn }}=\frac{h_{0}-\left(V_{v}^{*}+\left.\bar{w}_{z}\right|_{h_{F A}} ^{0}\right) T_{a p p}^{d e s}}{\left(V_{v}^{*}+\left.\bar{w}_{z}\right|_{h_{T I}} ^{0}-\left.\bar{w}_{z}\right|_{h_{F A}} ^{0}\right)}
$$


Using this corrected value of the final turn maneuver, allows onboard guidance unit to produce a more balanced control input $\dot{\psi}_{\text {opt }}(t)$.

In addition to correcting the time of the maneuver the optimization algorithm may include an additional varied parameter. To be more specific, we can replace the final condition (2) with the new one

$$
\mathbf{x}_{f}=\left[\left(V_{h}^{*}-W\right) T_{a p p}^{d e s} \cos \psi_{f},-\left(V_{h}^{*}-W\right) T_{a p p}^{d e s} \sin \psi_{f}, \psi_{f}\right]^{T}
$$

with the value of $\psi_{f}$ being variable. This allows cutting the final turn maneuver and gliding directly to the target with $\psi_{f} \neq-\pi$. Similar changes can be made for any wind model considered in the previous sections. Specifically, the final conditions of Eq.(19) can be substituted with

$$
\left[\begin{array}{c}
x \\
y
\end{array}\right]_{\tau=\tau_{f}}=\left[\begin{array}{c}
\left(\left(V_{h}^{*}-W_{G}\right) T_{a p p}^{d e s}-\frac{1}{2} K V_{v}^{*}\left(T_{a p p}^{d e s}\right)^{2}\right) \cos \psi_{f} \\
-\left(\left(V_{h}^{*}-W_{G}\right) T_{a p p}^{d e s}-\frac{1}{2} K V_{v}^{*}\left(T_{a p p}^{d e s}\right)^{2}\right) \sin \psi_{f}
\end{array}\right], \quad\left[\begin{array}{c}
\dot{x} \\
\dot{y}
\end{array}\right]_{\tau=\tau_{f}}=\left[\begin{array}{c}
V_{h}^{*} \cos \psi_{f}+W_{G}+K V_{v}^{*} T_{a p p}^{d e s} \\
V_{h}^{*} \sin \psi_{f}
\end{array}\right],
$$

Eq.(27) with

$$
\left[\begin{array}{c}
x \\
y
\end{array}\right]_{\tau=\tau_{f}}=\left[\begin{array}{c}
\left(\left(V_{h}^{*}+\alpha-\beta\right) T_{a p p}^{d e s}-\alpha T_{a p p}^{d e s} \ln \left(V_{v}^{*} T_{a p p}^{d e s}\right)\right) \cos \psi_{f} \\
-\left(\left(V_{h}^{*}+\alpha-\beta\right) T_{a p p}^{d e s}-\alpha T_{a p p}^{d e s} \ln \left(V_{v}^{*} T_{a p p}^{d e s}\right)\right) \sin \psi_{f}
\end{array}\right], \quad\left[\begin{array}{c}
\dot{x} \\
\dot{y}
\end{array}\right]_{\tau=\tau_{f}}=\left[\begin{array}{c}
V_{h}^{*} \cos \psi_{f}+\beta+a \ln \left(V_{v}^{*} T_{a p p}^{d e s}\right) \\
V_{h}^{*} \sin \psi_{f}
\end{array}\right],
$$

and Eq.(37) with

$$
\left[\begin{array}{l}
x \\
y
\end{array}\right]_{\tau=\tau_{f}}=\left[\begin{array}{c}
\sqrt{x_{f}^{2}+y_{f}^{2}} \cos \psi_{f} \\
-\sqrt{x_{f}^{2}+y_{f}^{2}} \sin \psi_{f}
\end{array}\right]
$$$$
\left[\begin{array}{c}
\dot{x} \\
\dot{y}
\end{array}\right]_{\tau=\tau_{f}}=\left[\begin{array}{l}
V_{h}^{*} \cos \psi_{f}+w_{x}(h) \\
V_{h}^{*} \sin \psi_{f}+w_{y}(h)
\end{array}\right]
$$

\section{Conclusion}

The direct-method-based approach to optimize the final-turn maneuver and mitigate all the errors of the previous phases of a guided flight of an aerodynamic decelerator system that was developed earlier for a simple onedimensional wind model can be modified to accommodate more complex models as well. Specifically, the paper presented modifications involving: i) variable (with altitude) downwind component of the wind based on no prior knowledge of the surface-layer winds or incorporating ground wind data provided by the target ground weather station on-line, ii) variable downwind and crosswind components, again based on online wind estimates at the current altitude and/or surface-layer wind magnitude and direction provided by the target ground weather station, and iii) variable 3D wind uplinked in real time by the preceding system. Even the original guidance and control architecture assures unprecedented into-the-wind touchdown accuracy of about 10m CEP with a maximum miss distance of $30 \mathrm{~m}$, demonstrated in over a hundred drops of the miniature autonomous parafoil delivery system Snowflake. Modifications presented in this paper will allow users to utilize more complex tactical scenarios, e.g. intentional landing with a substantial crosswind component or operating in the mountainous areas with significant variations in the vertical component of the wind, while preserving the superb touchdown accuracy.

\section{References}

${ }^{1}$ Slegers, N., and Yakimenko, O., “Terminal Guidance of Autonomous Parafoils in High Wind to Airspeed Ratios," Proceedings of the Institution of Mechanical Engineers, Part G: Journal of Aerospace Engineering, Vol. 225, No. 3, 2011, pp.336-346. doi:10.1243/09544100JAERO749.

${ }^{2}$ Yakimenko, O.A., Slegers, N.J., Bourakov, E.A., Hewgley, C.W., Jensen, R.P., Robinson, A.B., Malone, J.R., and Heidt, P.E., "Autonomous Aerial Payload Delivery System "Blizzard"," Proceedings of the $21^{\text {st }}$ AIAA Aerodynamic Decelerator Systems Technology Conference, Dublin, Ireland, May 23-26, 2011.

${ }^{3}$ Yakimenko, O., Slegers, N., and Tiaden, R., "Development and Testing of the Miniature Aerial Delivery System Snowflake," Proceedings of the $20^{\text {th }}$ AIAA Aerodynamic Decelerator Systems Technology Conference, Seattle, WA, May 4-7, 2009.

${ }^{4}$ Yakimenko, O., and Slegers, N., Initial Test of the Miniature Aerial Delivery System (Snowflake). TNT 08-03 Final Report, Naval Postgraduate School, Monterey, CA, 2008.

${ }^{5}$ Yakimenko, O., and Slegers, N., Autonomous UAV-Deployed Parafoil Delivery System “Snowflake”. TNT 10-03 Final Report, Naval Postgraduate School, Monterey, CA, 2010.

${ }^{6}$ Yakimenko, O., and Slegers, N., "Using Direct Methods for Terminal Guidance of Autonomous Aerial Delivery Systems," Proceedings of the European Control Conference, Budapest, Hungary, August 23-26, 2009. 
${ }^{7}$ Bourakov, E.A., Yakimenko, O.A., and Slegers, N.J., "Exploiting a GSM Network for Precise Payload Delivery," Proceedings of the 20 th AIAA Aerodynamic Decelerator Systems Technology Conference, WA, May 4-7 2009.

${ }^{8}$ Hewgley, C.W., and Yakimenko, O.A., "Improved Surface Layer Wind Modeling for Autonomous Parafoils in a Maritime Environment," Proceedings of the $21^{\text {st }}$ AIAA Aerodynamic Decelerator Systems Technology Conference, Dublin, Ireland, May 23-26, 2011.

${ }^{9}$ Hewgley, C.W., Yakimenko, O.A., and Slegers, N.J., "Shipboard Landing Challenges for Autonomous Parafoils," Proceedings of the $21^{\text {st }}$ AIAA Aerodynamic Decelerator Systems Technology Conference, Dublin, Ireland, May 23-26, 2011. 